\title{
Intracranial oligodendrogliomatosis
}

\author{
PHILIP V. BEST
}

\author{
From the Department of Pathology, University of Aberdeen
}

Of primary intracranial tumours, oligodendroglioma has been regarded as second only to medulloblastoma in its propensity to become disseminated through the ventricles and subarachnoid space (Blumenfeld and Gardner, 1945), but examples of this condition are nevertheless rare. It was recognized that spread may occur within the ventricular system (Cairns, 1929; Martin, 1931) before the discovery of the still less common dissemination in the leptomeninges (Greenfield and Robertson, 1933). Beck and Russell (1942) reported four cases of the latter type, in one of which the ventricles were not involved, and described the condition as oligodendrogliomatosis of the cerebrospinal pathway.

Blumenfeld and Gardner (1945) reviewed 11 cases from the literature in which there was spread of oligodendroglioma into the ventricles or meninges or both, excluding those with direct infiltration limited to the region of surgical operation, and added one of their own. In some of the cases reviewed, however, there was no definite evidence of metastatic dissemination through the cerebrospinal pathway, and the involvement of the leptomeninges or ventricles in these could be attributed to direct extension from the primary growth (Dickson, 1926; case 4 of Greenfield and Robertson, 1933; case 20 of Löwenberg and Waggoner, 1939).

Earnest, Kernohan, and Craig (1950) noted seeding via the cerebrospinal fluid in 14 of the 165 examples of oligodendroglioma seen in 30 years up to 1947 at the Mayo Clinic, but gave no details. Trowbridge and French (1952) reported another example in a man aged 38 years who had had three craniotomies over a period of six years for removal of the tumour. Berkheiser (1956) described two cases of oligodendroglioma in the young age group, the second of which was considered to show subarachnoid seeding and dissemination, although there is no mention of neoplastic deposits beyond the vicinity of the left frontal lobe where the primary was found.

The two cases which form the basis of the present report are examples of disseminated oligodendroglioma involving the ventricles and leptomeninges. The primary tumour had been partly removed some 15 months before death in the first case and surgical intervention may have promoted the spread of the tumour, but in the second case the dissemination was spontaneous. Additional features of interest in the second case are the early age of development of oligodendroglioma and the possible problems in differential diagnosis.

\section{CASE REPORTS}

CASE 1 T.M., a man aged 41 years, was admitted to the Neurosurgical Department of the Aberdeen Royal Infirmary on 18 September 1960 under the care of $\mathrm{Mr}$. R. J. A. Fraser. A large portion of a deep-seated, calcified tumour had been removed from the right posterior parietal region in October 1959 at another hospital and a diagnosis of oligodendroglioma was made histologically. His history before operation was one of increasing headache and defective memory for two months. Postoperatively his initial recovery was good and at the time of his discharge from hospital, apart from a persistent left homonymous field defect, there were no notable neurological abnormalities.

Three months before readmission to hospital he began to develop increasing weakness of the left arm and leg, mental confusion, and occasional periods of incontinence. He also complained of 'peculiar pains' in the left arm and leg, which were at times unbearable. On examination there was early papilloedema, more marked on the right than the left, and the other abnormal signs, all of which were on the left, were homonymous hemianopia, hemiparesis, pain of thalamic type on movement of the joints, absent reflexes except for a sluggish knee jerk, lack of coordination, and gross impairment of all forms of sensation, particularly severe below the level of T.11.

Further surgery was considered unjustifiable as the tumour was evidently so deeply situated, and the patient was treated symptomatically. The left-sided pain persisted and his general condition slowly deteriorated until his death on 31 January 1961.

Necropsy Necropsy was performed 22 hours after death. Apart from the presence of severe bronchopneumonia with widespread confluent consolidation, the significant pathological changes were confined to the head. Changes in the right parietal region attributable to the previous craniotomy were noted in the scalp and skull, which were otherwise normal. The brain weighed $1,350 \mathrm{~g}$. and was under moderately increased pressure, with bilateral tentorial herniation slightly more marked on the right than the left and slight herniation of the posterior part of the right cingulate gyrus beneath the falx. The dura was adherent to the surface of the brain in 
the right posterior parietal region beneath the site of the bone flap and when this was separated a large cavity was entered from which abundant clear fluid, resembling cerebrospinal fluid, escaped. The cavity was presumed to be the site from which tumour tissue had previously been removed and there was communication with the lateral ventricle. Induration could be felt in the cerebral substance anterior to the operative field as far as the temporal pole and medially across the corpus callosum to the medial part of the left hemisphere. The leptomeninges were slightly thickened over the anterior aspect of the pons and medulla but this was not a conspicuous feature.

The brain was fixed intact by suspension in formol saline and subsequent transection revealed the presence of extensive tumour infiltration which lacked clear definition, although the main mass was centred in and almost completely replaced the right thalamus (Fig. 1). The presence of infiltration anteriorly as far as the temporal pole and medially through the corpus callosum to the left cingulate gyrus and centrum semiovale was confirmed. In addition the right crus cerebri was invaded and there was a posterior tongue of tumour extending into the right occipital lobe below the posterior horn of the lateral ventricle. The large cavity already noted was lined by irregular greyish nodules of tumour and there were widespread metastases throughout the ventricular system including the cerebral aqueduct and fourth ventricle (Fig. 2). These deposits presented as soft, brownish, polypoid projections from the ependymal surface. The bulk of the infiltrative part of the tumour was firm and greyish, and gritty particles of calcareous material could be palpated in many areas. There were no cysts, assuming that the cavity in the right posterior parietal region could be attributed to surgery. There was only moderate hydrocephalus, which was surprising in view of the fact that the aqueduct appeared to be completely occluded by tumour tissue, but the ventricles probably collapsed due to escape of cerebrospinal fluid during removal of the brain.

Histological examination Representative blocks of tissue were taken after formalin fixation of the brain and sections were stained by haematoxylin and eosin, Van Gieson's method, Gordon and Sweet's silver impregnation method for reticulin, Mallory's phosphotungstic acid haematoxylin, Holzer's method for glial fibres, periodic acid-Schiff, or alcian blue for mucin.

The bulk of the infiltrative part of the tumour exhibits a typical oligodendrogliomatous structure (Fig. 3) with a fairly uniform cellular pattern of small rounded or ovoid nuclei, most of which have no recognizable surrounding cytoplasm but are suspended in a delicate reticular 'stroma' which gives a faintly positive reaction for mucin. The nuclei have a delicate chromatin pattern with a few chromatin granules but no definite nucleolus and mitotic figures are not identified. Scattered astrocytes are recognized and there is a scanty network of neuroglial fibres between the tumour cells. Where grey matter is involved surviving nerve cells can be seen in densely infiltrated areas, and in the cerebral cortex there is a notable tendency for the tumour cells to aggregate around neurones (Fig. 4). Subpial concentration of neoplastic tissue is also conspicuous in some areas (Fig. 4) even when the overlying leptomeninges are unaffected. There is moderate vascularity and most of the vessels are of small capillary type, but endothelial or adventitial proliferation is not observed. Calcium is deposited in many fields (Fig. 3) both as isolated calcospherites and as incrustations of vessel walls.

In contrast to these features, the metastatic tumour in the ventricles exhibits considerable pleomorphism (Fig. 5). Many cells indistinguishable from those described above are seen but these are outnumbered by large, bizarre cells with round, oval, indented or reniform nuclei containing one or two prominent nucleoli. Binucleate forms are common and there are occasional cells with three or four nuclei. Mitoticfigures are not infrequent. Themajority of these large cells have abundant eosinophilic cytoplasm, which is often vacuolated, but in some the cytoplasm is scanty or occasionally unrecognizable. Between these two extremes of cell structure intermediate forms can be identified. Degenerative changes are common, especially near the ventricular surface where many of the nuclei show pyknosis or karyorrhexis. Scanty marginal gliosis is noted in the deep parts of the metastatic tumour and subjacent brain, although the appearances here are in part the result of neoplastic infiltration of the subependymal fibrillary neuroglial layer. Occasional astrocytes can be recognized amongst the tumour cells but many of the latter so closely resemble reactive or neoplastic astrocyteso that the distinction is often difficult. Calcium is not observed in the metastatic tumour and vascular endothelial proliferation in some fields is conspicuous.

Meningeal infiltration by neoplastic cells is exceptiona $\bar{F}-\vec{c}$ and occurs only in the immediate vicinity of the intracerebral tumour. At such sites there is the same type ofo pleomorphic pattern as seen in the intraventricular $\vec{c}$ growths. Even where there is a dense subpial zone of oligodendroglioma, the overlying meninges are not usually involved. The meningeal thickening noted macroscopically at the base of the brain is due to fibrosis, and within it there are occasional groups of proliferated arachnoid cells which, however, are sometimes difficult to distinguish from tumour cells.

The tumour in this case arose in the vicinity of the right thalamus and dissemination through the ventricular system may have been spontaneous subsequent to penetration of the ependyma or may have been initiated by surgical interference. It is of considerable interest to note that, in spite of the extent and size of the ventricular metastases, the leptomeninges are involved to a very small extent and only by direct infiltration from subjacent intracerebral growth.

The bulk of the tumour has the classical histological features of an oligodendroglioma but in the ventricular and leptomeningeal growths there is considerable pleomorphism. It could be argued that dedifferentiation determined the propensity to dissemination, but such cellular pleomorphism has been observed in oligodendrogliomata without necessarily implying increased malignancy (Zülch, 1955), and 


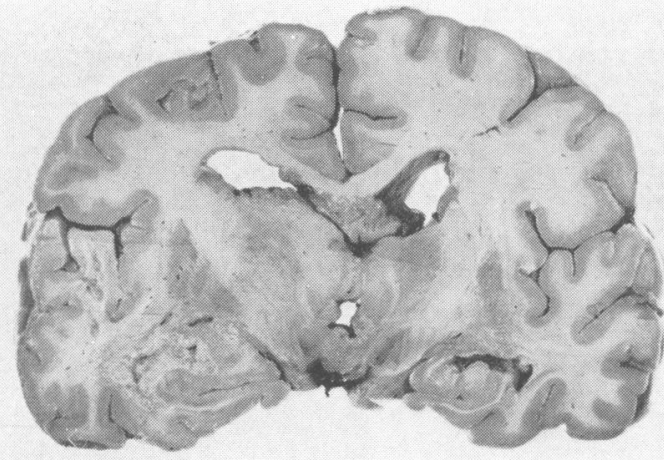

FIG. 1

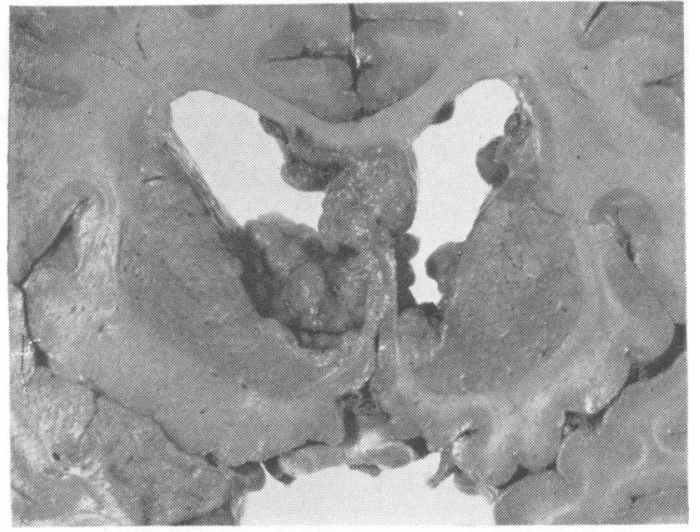

FIG. 2

FIG. 1. Case 1. Section of cerebral hemispheres, viewed from the front, to show tumour almost completely replacing the right thalamus and infiltrating the temporal lobe. Metastases can be seen in the walls of the lateral and third ventricles.

FIG. 2. Case 1. Section of cerebral hemispheres, viewed from the front, to show metastatic tumour nodules on the walls of the lateral ventricles. The anterior part of the primary growth can be seen in the right frontal and temporal lobes. The absence of meningeal thickening around the optic chiasma is to be noted.

FIG. 3. Case 1. A representative field from the infiltrative part of the primary tumour to show the uniform, 'honeycomb' architecture. Deposits of calcium are also seen. Periodic acid-Schiff, $\times 210$.

FIG. 4. Case 1. Cerebral cortex at the edge of the infiltrative growth to show subpial concentration of tumour cells both at the surface and around penetrating vessels. Perineuronal aggregates can also be seen and there is no invasion of the leptomeninges. Haematoxylin and eosin, $\times$ 110.

FIG. 5. Case 1. High-power view of a representative field from one of the ventricular metastases to illustrate the cellular pleomorphism. Periodic acid-Schiff, $\times 550$.

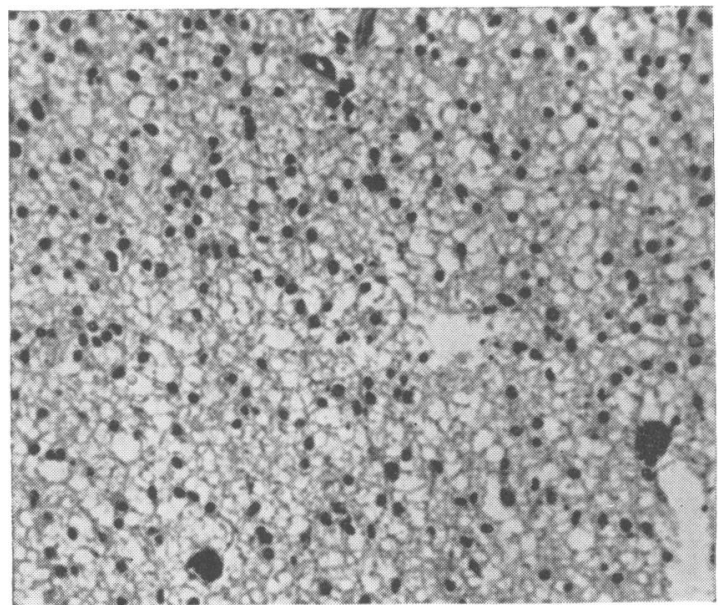

FIG. 3

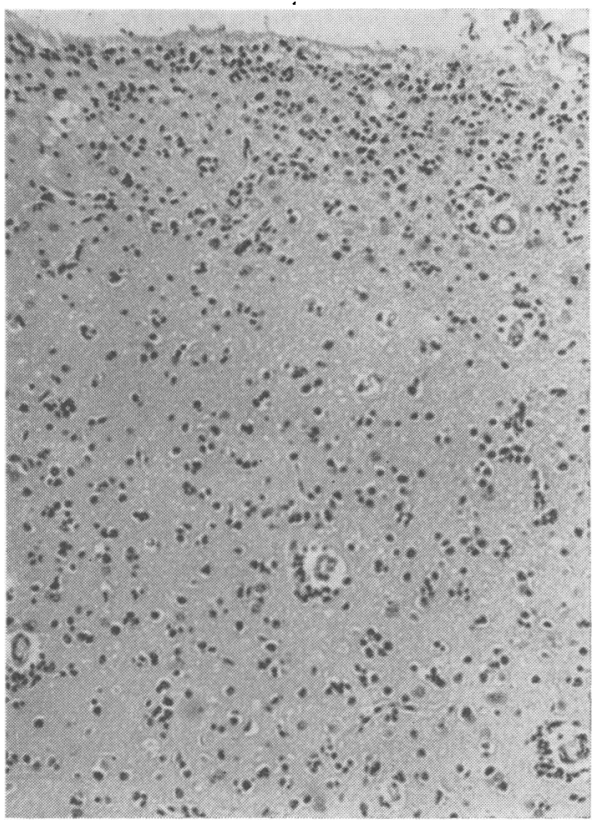

FIG. 4

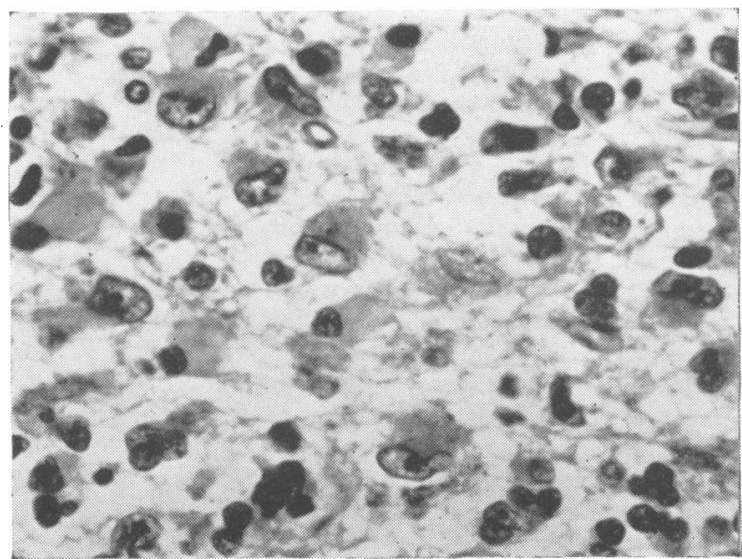

FIG. 5 
other surveys have shown that the behaviour of this type of glioma cannot be correlated with its microscopical structure (Shenkin, Grant, and Drew, 1947; Earnest et al., 1950).

CASE 2 I.F.G., a boy, $2 \frac{1}{2}$ years of age, was admitted to hospital on 9 May 1960, owing to the development of drowsiness and vomiting in the previous three weeks. His parents had noticed that from the time he could walk he had been very unsteady on his feet and tended to bump into objects. Five weeks before admission he had a short episode of vomiting followed by a period of ataxia, and two weeks later he fell off a chair, striking his forehead on the floor. Immediately afterwards he became drowsy, fell asleep, and vomited on waking. For the next few days he seemed quite well but then began to vomit again and this persisted, especially on waking in the mornings. At no time did he seem to suffer from headache. There was considerable loss of weight during the three weeks.

On examination he was drowsy and somewhat irritable. His temperature was $98.0^{\circ} \mathrm{F}$, pulse 96 per minute, and there was no sign of meningeal irritation. There was bilateral early papilloedema. No other abnormality was found in the cranial nerves, but on the right side of the body there was some decrease of tone and the reflexes were diminished. The plantar responses were flexor. The blood pressure was $98 / 58 \mathrm{~mm}$. $\mathrm{Hg}$ and there were no abnormal findings on general examination. The haemoglobin was $92 \%$, the total white blood cell count $6,650 /$ c. mm., and the erythrocyte sedimentation rate $9 \mathrm{~mm}$. in the first hour.

Left carotid angiography was carried out and the appearances were those of increased intracranial pressure with hydrocephalus. The evidence strongly suggested the presence of a posterior fossa tumour and on 10 May a posterior fossa craniotomy was performed by Mr. W. M. Nichols. The left lateral ventricle was first cannulated through an occipital burr hole and a large quantity of clear cerebrospinal fluid was released. During the posterior fossa operation profuse venous haemorrhage was encountered when the dura was being opened. Circulatory collapse occurred and after a considerable interval the operation was continued with the patient in the prone position, having been started in the usual sitting posture. It was found impossible to raise the blood pressure and the child's condition deteriorated steadily, The dura could only be opened after several clips had been applied to the edges of the incision, but it was then seen that there was marked arachnoid thickening in the vicinity of the cisterna magna which was much diminished in size and the foramen of Magendie greatly enlarged. The deterioration continued in spite of all attempts at resuscitation and the boy died on the operating table.

Necropsy Necrospy was carried out 22 hours after death. The spinal cord was not removed but otherwise a full post-mortem examination was made and the significant findings were confined to the head. There was no external abnormality apart from the recent posterior fossa craniotomy wound. There was an operative defect in the occipital bone slightly larger to the left than the

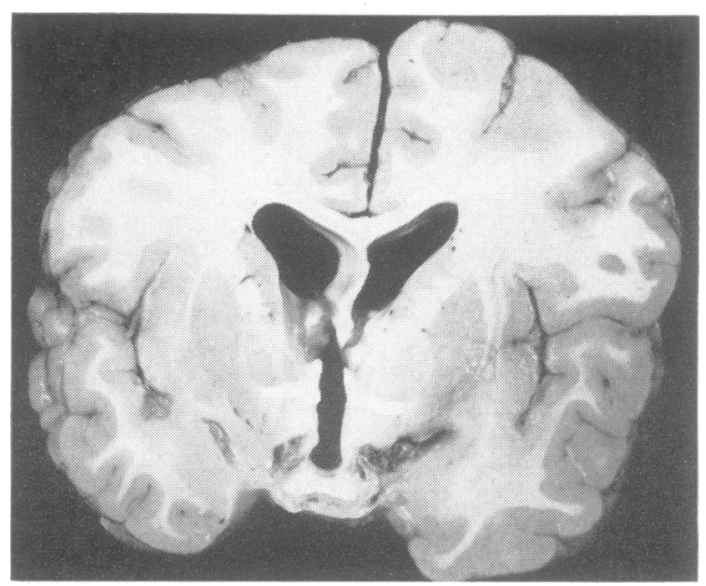

FIG. 6. Case 2. Section of cerebral hemispheres to show leptomeningeal thickening around the optic chiasma, mild symmetrical hydrocephalus, and the small tumour nodules on the ventricular walls.

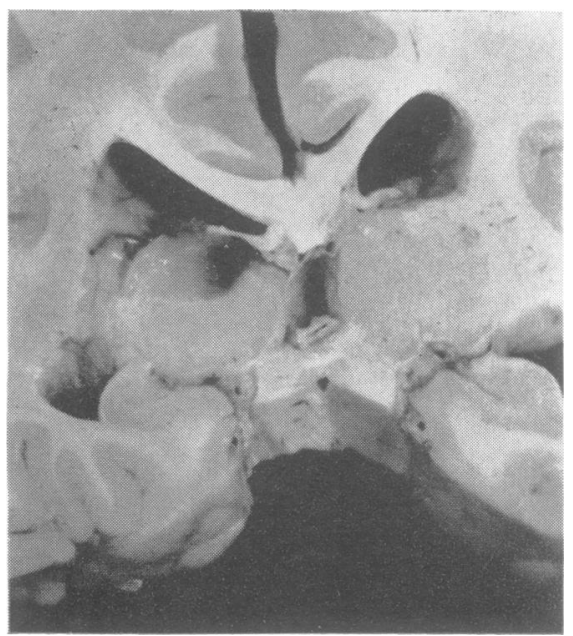

FIG. 7. Case 2. Posterior aspect of a section of brain at the level of the superior corpora quadrigemina to show partial replacement of the left pulvinar by greyish, haemorrhagic tumour.

right of the mid-line but the skull was otherwise normal. A considerable quantity of blood clot was present in the subdural space of the posterior fossa both in the operative field and anteriorly around the brain-stem, but blood had not penetrated above the tentorium. There was a congenital abnormality of the occipital venous sinuses, of which there was a pair, each approximately equal in size to the transverse sinuses, although the latter were somewhat narrower than normal. The left occipital sinus crossed the craniotomy site; silver clips were present in 


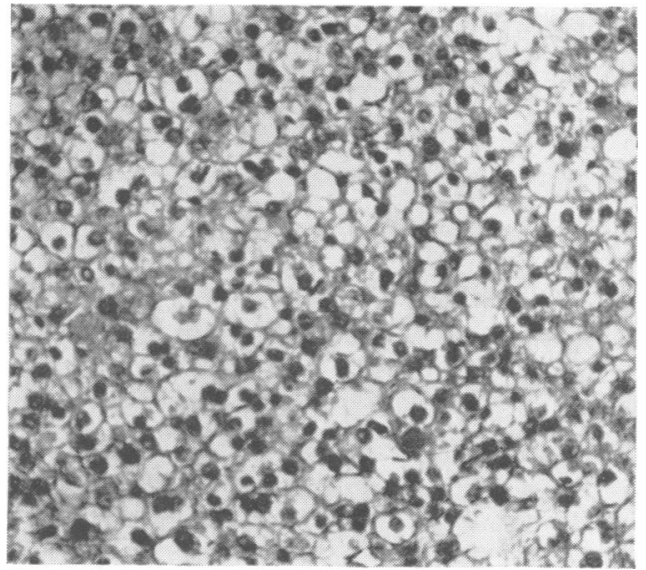

FIG. 8. Case 2. A representative field from thalamic tumour tissue to show uniform, 'honeycomb' architecture. Alcian blue, $\times 200$.

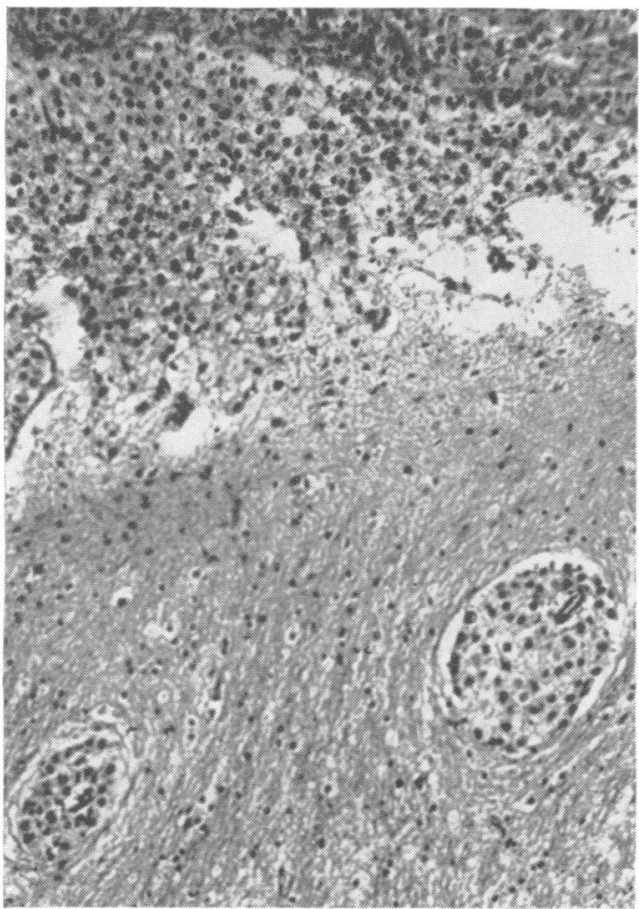

FIG. 9. Case 2. Postero-medial surface of thalamus adjacent to the primary tumour to show infiltration of leptomeninges with extension into Virchow-Robin spaces but no subpial concentration. Periodic acidSchiff, $\times 120$.

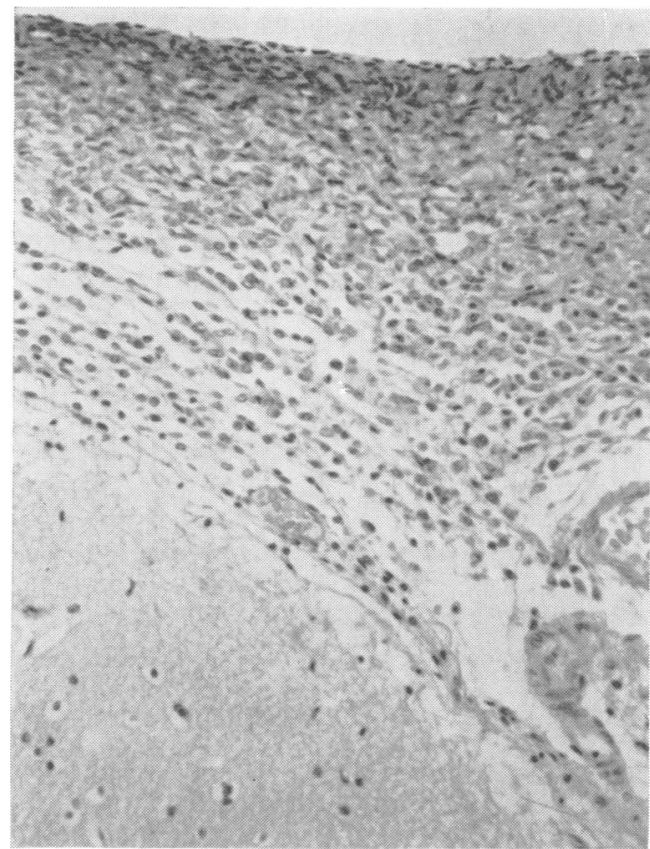

FIG. 10. Case 2. Section from the surface of gyrus rectus to show leptomeningeal thickening due to tumour infiltration accompanied by arachnoid cell and fibroblastic proliferation together with deposits of collagen. Haematoxylin-Van Gieson, $\times 155$.

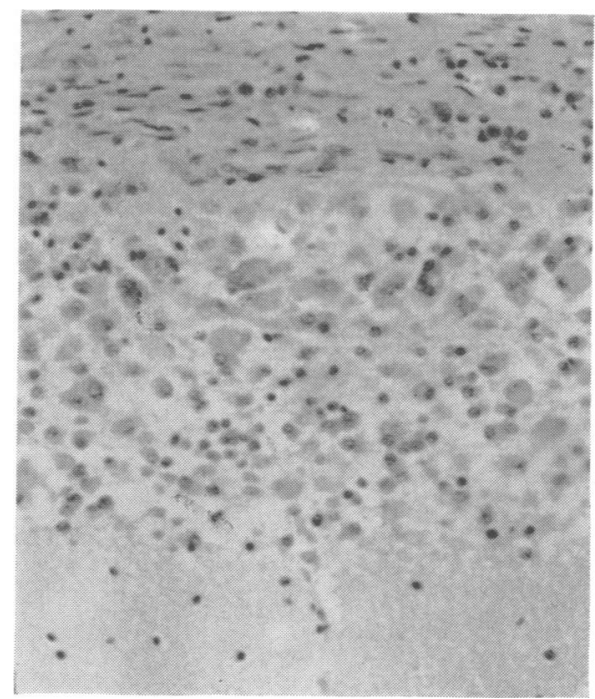

FIG. 11. Case 2. A field from cerebellar cortex to show superficial reactive astrocytic proliferation beneath the thickened meninges. Haemato$x y$ lin and eosin, $\times 190$. 
its wall and this was clearly the source of haemorrhage.

The brain was easily removed and no undue adhesion was encountered between the dura and the arachnoid. Moderate flattening of the gyri and narrowing of the sulci indicated the presence of raised intracranial pressure, but there was no significant tentorial or foraminal herniation. The leptomeninges were grossly thickened over the base of the brain, especially around the optic chiasma (Fig. 6) and on the anterior and lateral surfaces of the pons investing the cranial nerve roots. Similar but less conspicuous leptomeningeal thickening could be seen over the cerebellar hemispheres, particularly on the inferior aspect, then rapidly diminishing from the medulla to the upper cervical spinal cord where the meninges appeared normal. The thickening was visible also in the lateral fissures and a little distance onto the medial part of the inferior surfaces of the temporal and frontal lobes. Over the cerebral convexities the meninges appeared normal. The features closely resembled those of chronic basal meningitis, but tubercles could not be identified.

The brain was fixed intact by suspension in formol saline, and subsequent transection revealed the presence of mild symmetrical dilatation of the whole ventricular system. Numerous tiny, greyish nodules, up to $2 \mathrm{~mm}$. in diameter, were seen on the ependymal surfaces, especially of the lateral and fourth ventricles (Fig. 6). In the medial part of the pulvinar of the thalamus on each side, but with no visible intervening connexion, there was a soft greyish gelatinous area with a sharply defined edge, replacing thalamic tissue deep to the pial surface (Fig. 7). The lesion on the left, which measured $1.5 \times 1 \times 0.5 \mathrm{~cm}$., contained a focus of haemorrhage and just reached the ependyma of the lateral ventricle anteromedially; the one on the right was smaller $(0.5 \mathrm{~cm}$. in diameter), not haemorrhagic, and was situated slightly more posteriorly so that it did not reach the ventricular wall. The leptomeninges in the vicinity and the tela chorioidea were moderately thickened.

Histological examination Representative blocks of tissue were taken from all parts of the brain and were stained by the same methods as were used for case 1 .

Both thalamic lesions present a 'honeycomb' histological pattern (Fig. 8) which appears superficially similar to that of the infiltrative part of the tumour in case 1 , but on closer examination there are notable differences. The nuclear form is essentially the same but very occasional mitotic figures can be identified. Perineuronal aggregation and subpial concentration of cells are not observed and there is no calcium deposited. The 'stroma' gives a strongly positive reaction for mucin and there are scattered microcystic areas containing mucoid material. Surrounding thalamic tissue appears to be compressed and there is conspicuous marginal gliosis. Within the tumour itself there is also a fairly dense network of glial fibres. Tumour cells show little tendency to invade nervous tissue but extend a little distance into it as a sheath around blood vessels, filling the Virchow-Robin space and infiltrating the perivascular connective tissue (Fig. 9). It is confirmed that the tumour on the left reaches the ependyma of the lateral ventricle and that the one on the right does not, although the latter comes very close.

The leptomeninges overlying the thalamic tumour on each side are infiltrated by neoplastic cells and there is an associated fibroblastic proliferation together with abundant deposition of reticulin and collagen fibres. The thickened meninges elsewhere (Fig. 10) and the tela chorioidea of all the ventricles show similar features, although the density of fibrosis is variable. Tumour deposits are commonly found in parts of the leptomeninges that appeared to be normal macroscopically, and some of these, presumably relatively recent, are accompanied by little or no deposited reticulin or collagen. Intermediate degrees of fibrosis can be found from this up to dense collagenous thickening such as that around the base of the brain, and in these areas oligodendrogliomatous cells are often difficult to identify. Proliferation of arachnoid cells is commonly encountered and these are frequently very difficult to distinguish from neoplastic cells. Inflammatory cells of any type are rare.

Superficial gliosis is usually present immediately beneath the thickened meninges and this varies from a slight increase in the thickness of the subpial glial fibre layer up to a dense fibrillary gliosis. In occasional foci there is an associated superficial astrocytic proliferation, and multinucleate, reactive forms are common in these fields (Fig. 11).

The ventricular nodules consist of small tumour deposits which project into the cavity of the ventricle at the site of a breach in the ependyma. The structure iș $\omega$ very similar to that of the thalamic tumours and there is in sometimes a superficial layer of pyknotic cells. Invasio $\$$ does not occur deep to the subependymal glial layer, but 응 lateral extension occasionally produces a broader oligodendrogliomatous plaque with some underminint of the ependyma at the edges.

The sequence of events in this case seems to have been first the development of a thalamic oligodendroglioma, or possibly one on each side, followed by dissemination through the cerebrospinal fluid pathway. The tumour on the left is slightly larger than that on the right and may have been the primary site of neoplasia, spread to the opposite side having occurred through the intervening leptomeninges. $\vec{\overline{ }}$ Tumour cells probably entered the left lateral ventricle through a breach in the ependyma over the $\bar{\partial}$ thalamus and were thence carried by the cerebro- 7 spinal fluid to the other ventricles and to the subarachnoid space. The possible alternative route of access of these cells to the cerebrospinal fluid pathway may have been by direct extension to the leptomeninges and subarachnoid space in the pineal region with subsequent retrograde spread into the ventricles favoured by the presence of communicating hydrocephalus.

The gliomatous elements in the meninges induced $\frac{T}{O}$ a deposition of reticulin and collagen, associated with arachnoid cellular proliferation, and in many $\mathrm{N}$ areas this reaction is of such a degree as to over- $N$ shadow the neoplasia. The meningeal thickening at $\mathrm{N}$ the level of the tentorium was clearly the cause of 
hydrocephalus. Reactive gliosis in relation to the tumour was also a notable feature.

Cellular pleomorphism and mitotic activity were inconspicuous throughout. Characteristics of malignancy as judged from histological appearances were thus lacking, in spite of the extent of dissemination and in contrast to the structure of the metastases in case 1.

\section{DISCUSSION}

The diagnosis in case 1 was straightforward but in case 2 there were difficulties. Macroscopically, before the brain was cut, the appearances closely resembled those of chronic arachnoiditis although there was nothing in the clinical history to suggest the presence of meningitis. This similarity endorses the view expressed by Beck and Russell (1942) that cases of the same type may easily escape recognition if a detailed examination of the central nervous system is not made. This is especially true if, as in case 2, the primary tumour is very small: the diagnosis would almost certainly have been missed in this child if it had depended solely upon examination of a small meningeal biopsy specimen.

The possibility of diffuse meningiomatosis entered into the differential diagnosis of case 2 and was favoured at first. The age of onset is exceptional for oligodendroglioma: the child died at the age of $2 \frac{1}{2}$ years and symptoms had been present at least from the time he could walk. A considerable proportion of the reported cases of diffuse meningiomatosis have been in young children (Bailey, 1929; Harbitz, 1935; Lichtenstein and Ettleson, 1937; Weinberger, 1940; Black and Kernohan, 1950). The main histological feature which led to difficulty in diagnosis was the conspicuous arachnoid cell proliferation seen in many areas which often completely obscured the presence of oligodendrogliomatous elements, and in other fields the distinction between the two was hardly possible. The other misleading appearance was the considerable glial fibre component of the thalamic lesions. It was thought conceivable that these lesions constituted an unusual gliomatous reaction to the presence of meningeal neoplasia, as varying degrees of subpial gliosis could be found elsewhere. 'Glial hernias' in a case of diffuse mesothelioma of the meninges were reported by Lichtenstein and Ettleson (1937) and meningiomata are known sometimes to be associated with an adjacent gliotic or even gliomatous reaction (Russell and Rubinstein, 1959). The ependymal granulations may have represented a similar reaction to the presence of metastatic tumour, although such lesions in Lichtenstein and Ettleson's case were attributed by them to glial hernias through congenital defects. The occurrence of cells of astrocytic type is, however, well known in oligodendrogliomata, and the features in case 2 are more readily explained on this basis. The appearances also correspond closely with those of some of the previously reported cases of oligodendrogliomatosis.

The cause of the mesodermal and glial reaction to the presence of the oligodendroglioma is a matter for speculation but it seems probable that the mucoid material, which is such a notable component of the tumour, may be partly responsible. A mesodermal reaction is, however, common in relation to tumours not associated with the formation of mucoid substance.

In view of the extent of meningeal dissemination of the oligodendroglioma in case 2 , it is of particular interest to note that this was minimal in case 1 in spite of the fact that conditions seemed to be eminently favourable for its occurrence. Seeding had occurred throughout the ventricular system in case 1 so that it must be assumed that viable neoplastic cells were being carried in the cerebrospinal fluid. Perhaps mechanical factors operated to filter off these cells at the foramina of Luschka and Magendie, but even in the few areas where direct invasion of the leptomeninges was found over the tumour there was little tendency for spread to occur in the subarachnoid space. This difference in behaviour of the tumour in the two cases is well illustrated by a comparison of the perivascular infiltrate in Figures 4 and 9. It seems more likely that for some reason the growth of the two tumours required different environmental conditions. To judge from the cases reported in the literature spread within the ventricular system is more common than it is through the subarachnoid space. Shenkin et al. (1947), for example, noted several instances of seeding on the ependyma of the ventricles in their 25 cases of oligodendroglioma but not one of meningeal dissemination. The tendency for spread of oligodendroglioma to occur through the cerebrospinal pathway also seems frequently to be precipitated or accelerated by surgical intervention.

\section{SUMMARY}

Two examples of disseminated intracranial oligodendroglioma are described, one in a 41-year-old man and the other in a child aged $2 \frac{1}{2}$ years. In the adult the metastases were almost entirely confined to the ventricular system and probably developed post-operatively, whereas in the child spread of the tumour occurred spontaneously and was predominantly in the leptomeninges. Possible reasons for this difference in behaviour are briefly discussed. 
The mesodermal reaction in the leptomeninges of the child was conspicuous and gave rise to problems in the differential diagnosis.

I am greatly indebted to Professor D. S. Russell for her advice with regard to the diagnosis of case 2, to Professor J. S. Young for his encouragment and criticism in the preparation of this paper, to Mr. W. M. Nichols and Mr. R. J. A. Fraser for permission to publish clinical details, and to Mr. W. Topp for the photographs.

\section{REFERENCES}

Bailey, P. (1929). Arch. Surg., 18, 1359.

Beck, D. J. K., and Russell, D. S. (1942). Brain, 65, 352.

Berkheiser, S. W. (1956). J. Neurosurg., 13, 170.

Black, B. K., and Kernohan, J. W. (1950). Cancer (Philad.), 3, 805.

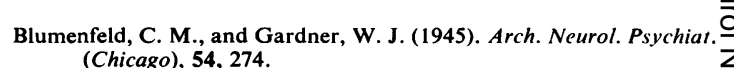

Cairns, H. (1929). Spec. Rep. Ser. med. Res. Coun. (Lond.), No. 125 p. 15.

Dickson, W. E. C. (1926). Brain, 49, 578.

Earnest, F., Kernohan, J. W., and Craig, W. M. (1950). Arch. Neuro:: Psychiat. (Chicago), 63, 964.

Greenfield, J. G., and Robertson, E. G. (1933). Brain, 56, 247.

Harbitz, H. F. (1935). Acta Path. microbiol. scand., 12, 24.

Lichtenstein, B. W., and Ettleson, A. (1937). Arch. Path. (Chicago) 24, 497.

Löwenberg, K., and Waggoner, R. W. (1939). Arch. Neurol. Psychiat. (Chicago), 42, 842.

Martin, J. P. (1931). Brain, 54, 330.

Russell, D. S., and Rubinstein, L. J. (1959). Pathology of Tumours of the Nervous System, p. 61. Arnold, London.

Shenkin, H. A., Grant, F. C., and Drew, J. H. (1947). Arch. Neurol. Psychiat. (Chicago), 58, 710.

Trowbridge, W. V., and French, J. D. (1952). J. Neurosurg., 9, 643.

Weinberger, L. M. (1940). Amer. J. Cancer, 38, 1.

Zülch, K. J. (1955). Excerpta med. (Amst.), Sect. VIII, 8, 816.

\section{The April 1963 Issue}

\section{THE APRIL 1963 ISSUE CONTAINS THE FOLLOWING PAPERS:-}

The relationship between enzyme activity and neuroglia in plaques of multiple sclerosis $\mathrm{M}$. $\mathbf{Z}$. $\mathrm{M}$. IBRAHIM and C. W. M. ADAMS

A trial of therapy by nucleosides and nucleotides in muscular dystrophy w. H. S. THOMSON and KENNETH E. GUEST

A biochemical study of Huntington's chorea F. E. KENYON and S. M. HARDY

Defect of memorizing of hippocampal mammillary origin: A review J. BARBIZET

The refractory and supernormal periods of the human median nerve R. W. GILLIATT and R. G. WILLISON

The relative importance of the gastrocnemius and soleus muscles in the ankle jerk of man RAYMOND LEVY

Dermal sensitivity tests in multiple sclerosis RICHARD T. JOHNSON and HENRY MILLER
A follow-up study of surgery in temporal low epilepsy MURRAY A. FALCONER and EUSTACE $\vec{A}$. SERAFETINIDES

The spinal canal in cervical spondylosis CHRISPIN and F. LEES

A. R.

Paraplegia due to spinal epidural haematoma B. H. DAWSON

Bilateral saccular aneurysms of the internal carotid artery in the cavernous sinus CHARLES B. WILSON and FAY $\mathrm{K}$. MYERS

The morphology of Marinesco bodies (paranucleolar 0 corpuscles) in the melanin-pigmented nuclei of the brain-stem P. YUEN and D. W. BAXTER

Patterns of insomnia in depressive states J. M. 응 HINTON

Book reviews

Copies are still available and may be obtained from the PUBLISHING MANAGER, BRITISH MEDICAL ASSOCIATION, TAVISTOCK SQUARE, W.C.I., price $18 \mathrm{~s}$. 6D. 